\title{
Label-free and fluorescence biosensing platform using one dimensional photonic crystal chips
}

\author{
F. Michelotti ${ }^{\mathrm{a}}$, S. Schmieder ${ }^{\mathrm{b}}$, A. Anopchenko ${ }^{\mathrm{a}}$, P. Munzert $^{\mathrm{c}}$, A. Sinibaldi ${ }^{\mathrm{a}}$, R. Chandrawati ${ }^{\mathrm{d}}$, \\ S. Rana ${ }^{\mathrm{d}}$, F. Sonntag ${ }^{\mathrm{b}}$, A. Occhicone ${ }^{\mathrm{a}}$, L. Napione ${ }^{\mathrm{e}}$, M. M. Stevens ${ }^{\mathrm{d}}$, E. Maillart ${ }^{\mathrm{f}}$, F.-E. Hibtif \\ C. Frydman ${ }^{\mathrm{f}}$, and N. Danz \\ ${ }^{a}$ SAPIENZA University of Roma, Department of Basic and Applied Sciences for Engineering, Via \\ A. Scarpa, 1600161 Roma, Italy; ${ }^{b}$ Fraunhofer Institute for Material and Beam Technology IWS, \\ Dresden, Germany; ${ }^{\mathrm{C}}$ Fraunhofer Institute for Applied Optics and Precision Engineering IOF, Jena, \\ Germany; ${ }^{d}$ Imperial College London, Department of Materials, London, UK; ${ }^{\mathrm{e}}$ University of Torino, \\ Department of Oncology, Cancer Institute of Candiolo, Candiolo, Italy; ${ }^{\mathrm{f}}$ Horiba Scientific, Palaiseau, \\ France
}

\begin{abstract}
The increasing demand for early detection of diseases drives the efforts to develop more and more sensitive techniques to detect biomarkers in extremely low concentrations. Electromagnetic modes at the surface of one dimensional photonic crystals, usually called Bloch surface waves, were demonstrated to enhance the resolution and constitute an attractive alternative to surface plasmon polariton optical biosensors. We report on the development of Bloch surface wave biochips operating in both label-free and fluorescence modes and demonstrate their use in ovalbumin recognition assays.
\end{abstract}

Keywords: optical biosensing, label-free biosensors, photonic crystals, Bloch surface waves, biophotonics, ovalbumin

\section{INTRODUCTION}

Recently, Bloch surface waves (BSW) propagating at the surface of truncated one dimensional photonic crystals ${ }^{1,2}(1 \mathrm{D}-$ PC) have been suggested for label-free biosensors based on optical configurations similar to those used in surface plasmon resonance $(\mathrm{SPR})^{3,4}$, including angularly resolved detection modes. BSW biosensors feature very low angular resonance widths compared to that of SPR, due to the low losses in the dielectric stacks ${ }^{5}$. Furthermore, the optimization of the 1DPC stack, in terms of geometry and materials, offers the possibility to improve the label-free operation, by tuning the resonance position and width ${ }^{6,7}$.

When targeting analytes at low concentrations, label-free biosensing may suffer from less-than-ideal suppression or referencing of unspecific effects and yield a non-adequate limit of detection (LoD). Coupling, in a single instrument, the label-free operation to a complementary detection system relying on the detection of fluorescent labels leads to a multimodal analysis and can decrease the LoD. The strong field localization and enhancement at the 1D-PC surface, and the absence of quenching, favor utilizing BSW coupled fluorescence emission ${ }^{8,9}$. In such a coupled configuration, the BSW properties are exploited in two ways. First, the BSW angular resonance position is tracked as a label-free signal. Second, the BSW coupled emission yields a specific fluorescence detection whenever a labelling step is performed.

We report on the development and results of a combined label-free and fluorescence biosensing platform utilizing disposable biochips. As described in our previous works ${ }^{10,11}$, a new optical system has been designed and fabricated, combining SPR-like angularly resolved resonance and the detection of fluorescent labels. All analysis can be performed in a parallel manner for multiple spots that are arranged as a one-dimensional array inside a microfluidic channel. In order to enable for later on disposable use, injection molded polymer substrates have been prepared and coated with low loss 1D-PC. Here we demonstrate the use of this multimode platform in ovalbumin recognition assays.

\section{BSW BIOCHIPS}

BSW are localized at the interface between a finite 1D-PC and an external medium by Bragg reflection and total internal reflection (TIR) on the two sides of the interface, respectively ${ }^{4}$. Like in SPR, the excitation of a BSW at a given wavelength 
$\lambda_{0}$ can be obtained by a prism coupler in the Kretschmann-Raether configuration ${ }^{12}$. The BSW excitation appears as a dip in the angular reflectance spectrum, whose position is very sensitive to refractive index perturbations at the interface and this attractive property can be used for bio-sensing ${ }^{13}$.

The fabrication of the 1D-PC was carried out by plasma ion assisted evaporation of dielectric materials (PIAD) under high vacuum conditions. PIAD enables the fabrication of dielectric multilayers with low absorption losses and the possibility to deposit on plastic substrates ${ }^{14}$.

As substrates for the 1D-PC deposition we used purposely developed and manufactured injection molded polymer chips made out of TOPAS $^{\circledR, 11}$. These comprise a prism shaped cross section in order to excite the BSW in Kretschmann configuration. Additionally, a two-component flow cell has been molded. It consists of a hard polymer cover that can be clicked by hand onto the chip. Inside this cover an elastomer defines a straight micro channel of $800 \mu \mathrm{m} \times 100 \mu \mathrm{m}$ cross section as well as perpendicularly aligned fluid connectors.

We used $\mathrm{SiO}_{2}, \mathrm{Ta}_{2} \mathrm{O}_{5}$ and $\mathrm{TiO}_{2}$ as dielectric materials for the 1D-PC deposition by PIAD. The complex refractive indices of such dielectric materials were determined either by reflection/transmission spectroscopy on single supported thin films or by ellipsometry on test multilayers sustaining BSW to be $\mathrm{n}_{\mathrm{SiO} 2}=1.474+\mathrm{i} 5 \times 10^{-6}, \mathrm{n}_{\mathrm{Ta} 2 \mathrm{O} 5}=2.160+\mathrm{i} 5 \times 10^{-5}$, and $\mathrm{n}_{\mathrm{TiO} 2}$ $=2.28+\mathrm{i} 1.8 \times 10^{-3}$ at the chosen wavelength of operation $\lambda_{0}=670 \mathrm{~nm}^{15}$. Using such values enables one to optimize the stacks for operation in water environment according to the considerations published elsewhere ${ }^{7}$. The final structure of the stack is shown in Figure 1(a), where the transverse intensity distribution for the BSW at $\lambda_{0}$ is also shown. In this stack, a $20 \mathrm{~nm}$ thin $\mathrm{TiO}_{2}$ layer is combined with a periodic $\mathrm{SiO}_{2} / \mathrm{Ta}_{2} \mathrm{O}_{5}$ stack of $275 \mathrm{~nm}$ and $120 \mathrm{~nm}$ thicknesses, respectively. In order to work with silane-based surface functionalization protocols, the stack is covered with a $20 \mathrm{~nm}$ thin $\mathrm{SiO}_{2}$ layer.

Figure 1(b) illustrates the dispersion of the resonance in the wavelength-angle plane. Two surface modes exhibiting different polarization are apparent. The TE resonance has been optimized for label-free sensing, but the stack design supports a rather wide and shallow TM mode at lower angles (wavelengths). Here only the TE mode is utilized for labelfree operation, whereas both TE and TM modes contribute to the fluorescence operation.

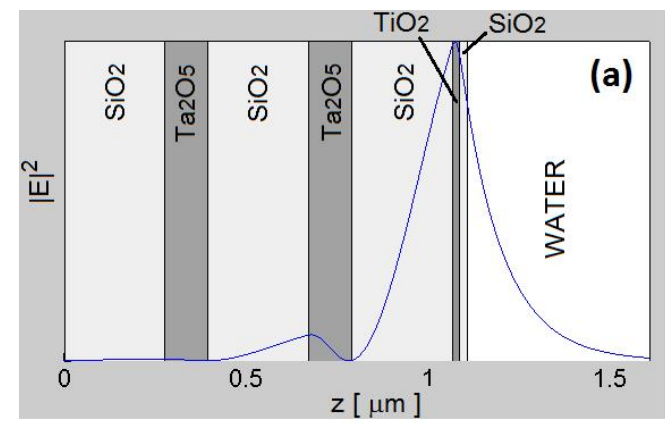

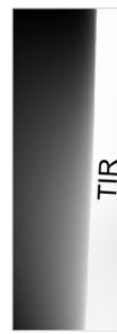

$\begin{array}{llllllll}58 & 60 & 62 & 64 & 66 & 68 & 70\end{array}$ Angle in substrate $\left[{ }^{\circ}\right] \longrightarrow$

(b)

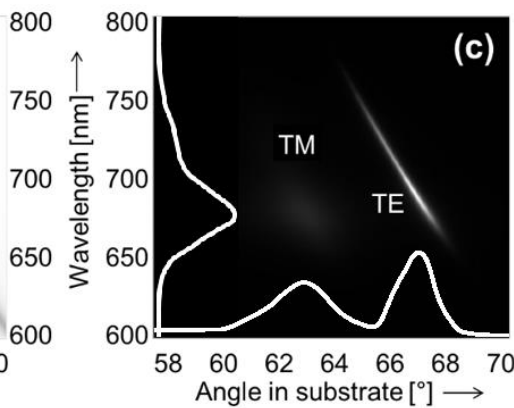

Figure 1. (a) Sketch of the 1D-PC operating in water environment with TE intensity distribution at $\lambda_{0}$. The substrate is made of TOPAS ${ }^{\circledR}$ polymer. (b) Grey scale plot of angularly and spectrally resolved reflectivity of the stack when illuminated from the substrate. (c) Emission pattern of DyLight650 dye molecules located on top of the thin film stack and plotted in grey scale representation. The labels "TE" and "TM" indicate the polarization of the resonances and "TIR" the dispersion of the total internal reflection edge. Along the vertical and horizontal axes the emission spectra of DyLight 650 either in water solution or on top of the biochip (in water) are superimposed, respectively.

Fluorescence emission by randomly oriented dye labels located on top of the 1D-PC is simulated rigorously by means of a transfer-matrix algorithm ${ }^{16}$ frequently applied for the modelling of Organic LED ${ }^{17}$. In Figure $1(\mathrm{c})$ we show the results when the emission spectrum of the DyLight650 dye (Abcam, UK) is assumed ${ }^{18}$. The fluorescence emission is coupled preferentially into the surface modes, leading to a resonant enhancement ${ }^{8}$ and to the possibility to collect it in a narrow angular range. Fluorescence is emitted according to the dispersion of the BSW resonance, therefore each wavelength component is emitted at a slightly different angle as defined by the 1D-PC dispersion.

\section{OPTICAL CONFIGURATION OF THE DETECTION PLATFORM}

In Figure 2 we show a simplified scheme of the optical system for BSW-based biosensing. For label-free operation (Figure $2 \mathrm{a})$, a collimated light beam (B) is focused by a cylindrical lens $(\mathrm{C} 1)$ into the biochip. The prismatic shape of the TOPAS ( 
substrate permits to operate above the critical angle for TIR at the biochip/external medium interface. A laser diode at $\lambda_{0}$ is used to achieve proper resolution of the narrow angular BSW resonances. The detection optical system features an extended CCD detector (Apogee Ascent equipped with Sony ICX814 CCD chip) to obtain 3388 (angular axes) and 2712 (position axes) pixels readout.

The cylindrical Fourier lens (F) permits to image the angular reflectance spectrum on the CCD detector. The angular field of view is determined by the cylindrical Fourier lens to be approximately 2.9 deg. Full discretization of the CCD chip (no binning) is exploited in order to achieve proper sampling of the resonance.

The cylindrical focusing approach creates an illuminated line along the direction $\mathrm{x}$ on the surface. Several measurement spots can be arranged in a row along the illuminated line. Parallel readout of the spots is achieved by using a cylindrical detection optical system that consists of objective (C2) and tube lens (C3) systems in order to image the direction $\mathrm{x}$ onto one coordinate of a CCD detector. The lateral field of view (coordinate $\mathrm{x}$ in Figure 2) is approximately $6 \mathrm{~mm}$; 8 pixels binning is applied, complying to the spatial resolution of lateral imaging. The design of the optical detection system ensures that the same angular range is observed for all spots along the illuminated region on the sensor surface.

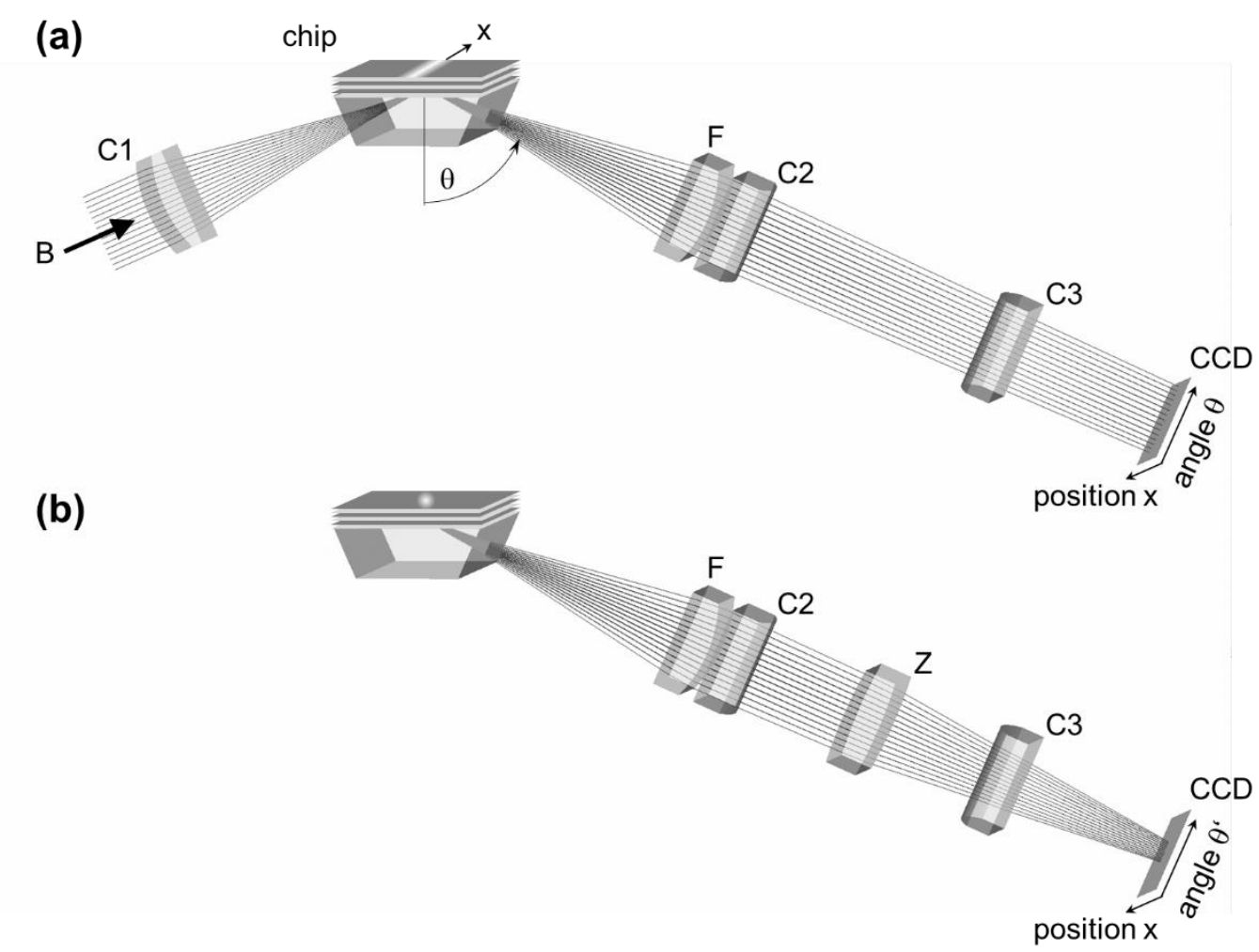

Figure 2. Simplified view of the BSW-based optical system in label-free (a) and fluorescence (b) modes (from Ref.11). In the label-free mode (a), a collimated beam (B) is focused by means of a cylindrical lens (C1) into the chip to create an illuminated line. A Fourier lens (F) images the angular spectrum of light reflected at the sensor surface onto the CCD detector. Cylindrical objective (C2) and tube lens systems (C3) image the lateral coordinate onto one axis of the CCD detector. An additional cylindrical lens $(\mathrm{Z})$ is introduced for fluorescence detection (b) to increase the angular detection range.

The fluorescence operation is based on properly selected dye labels which emit around $\lambda_{0}$, after excitation at a lower wavelength. Suitable dyes are: DyLight650 from Abcam, Alexa 647 from Thermo-Fisher, and Cy5. Dyes detection follows the approach described recently ${ }^{9}$ and is based on $\lambda_{\text {exc }}=635 \mathrm{~nm}$ illumination. Spatial imaging and binning are identical to the label-free case. However, Figure 1c illustrates that the angular range used for the label-free operation (2.9 deg) would be too small to detect the full angular emission spectrum for both TE and TM polarizations. Therefore, an additional cylindrical lens (Z) in Figure 2(b) is introduced into the light path in order to increase the angular field of view. The limited angular resolution associated with this approach is acceptable, and consequently angular binning of 8 pixels is utilized in fluorescence detection mode. 
Filter elements for selecting a certain polarization and/or spectral range are arranged in-between the cylindrical objective (C2) and tube lens (C3) systems. The spectral fluorescence emission filter (Chroma 685/70) is fixed in the detection optics because the label-free operation wavelength $\lambda_{0}$ is well within the fluorescence emission band and transmits such element.

\section{ASSAY FOR OVALBUMIN DETECTION}

In order to test the performance of the biosensing platform we developed, we carried out assays for the detection of ovalbumin (OVA) at several different concentrations in D-PBS 1X buffer. Figure 3 depicts the steps of the protocol we used in this detection assay. We briefly describe the protocol before discussing each step in detail in the following.

The protocol starts with a preparation phase beginning from a bare BSW chip constituted by a 1D-PC with top $\mathrm{SiO}_{2}$ layer deposited on a TOPAS ${ }^{\circledR}$ substrate. In the test experiments reported here, each biochip surface is divided into two areas defined as signal and reference regions. In the two regions we immobilize either the specific antibody anti-ovalbumin (aOVA) or a non-specific antibody anti-Angiopoietin 2 (a-Ang2), respectively. These two regions are illuminated simultaneously by the optical system and permit subtracting parasitic contributions taking place during the assay. After preparation, the biochip is topped with the hard polymer cover and its elastomer contact element, defining the straight micro channel passing over the two measurement regions. Then the biochip is mounted in the optical system (clicked in) and is used for the label-free and fluorescence assays.

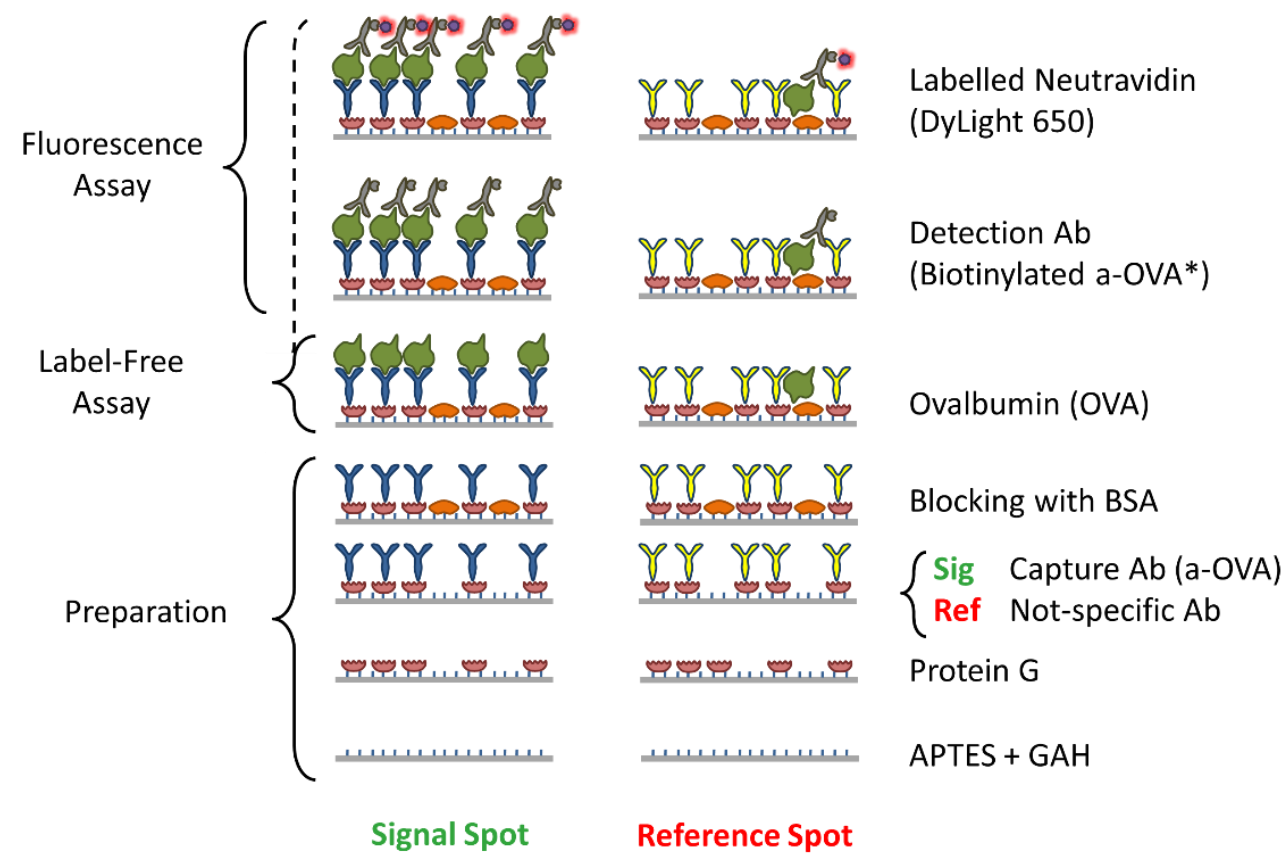

Figure 3 - Protocol used for the ovalbumin detection assays. Each BSW chip is divided into two areas defined as signal and reference spots. Capture antibodies and non-specific antibodies are conjugated onto the chip surfaces (signal spot and reference spot, respectively) by silane-based conjugation chemistry. The presence of ovalbumin can be detected based on label-free sensing. Subsequent addition of detection antibodies and fluorescent dyes enables detection at fluorescence mode.

\subsection{Biochip preparation}

In order to obtain a clean silica surface of the biochips, the bare 1D-PC surfaces were exposed to a piranha solution (3:1 mixture of sulfuric acid and 30\% hydrogen peroxide) for 10 minutes. Subsequently, the substrates were carefully rinsed with de-ionized (DI) water and dried under a stream of nitrogen gas. The cleaned biochips were then immersed into a $2 \%$ (v/v) solution of APTES ((3-Aminopropyl)triethoxysilane from Sigma-Aldrich) in ethanol/water (95:5 v/v) mixture at ambient temperature for $1 \mathrm{~h}$. Afterwards the chips were removed from the solution, sonicated ( $30 \mathrm{~s})$, rinsed with ethanol, and dried on a hot plate at $80^{\circ} \mathrm{C}$ for $1 \mathrm{~h}$. 
The specific (a-OVA) and non-specific (a-Ang2) antibodies immobilization starts with the coupling of the silane with $1 \%$ (v/v) glutaraldehyde (GAH) (Sigma-Aldrich) solution in $100 \mathrm{mM}$ sodium bicarbonate buffer (pH 8.5) in the presence of $0.1 \mathrm{mM} \mathrm{NaCNBH} 3$ (sodium cyanoborohydride, Sigma-Aldrich) for $1 \mathrm{~h}$ at room temperature. A further sonication in DI water was performed prior to thoroughly rinsing the biochips with DI water. A $0.5 \mathrm{mg} / \mathrm{ml}(40 \mu \mathrm{l})$ solution of Protein G $(\mathrm{PtG})$ (Thermo Scientific) in sodium bicarbonate buffer was dropped on the sensing area, allowed to react for $1 \mathrm{~h}$ at room temperature, and then removed by washing with D-PBS $1 \mathrm{X}$. The role of PtG is to properly orient the capture antibodies and maximize their recognition efficiency.

After PtG immobilization we proceeded with the definition of the two sensitive regions. These are separated from each other by a permanent marker that is used to draw a line in the middle of the biochip channel, that works as a hydrophobic barrier for the antibodies solutions during immobilization. We incubated overnight at $4{ }^{\circ} \mathrm{C}$ a-Ova $(8 \mu \mathrm{M}, 20 \mu \mathrm{l})$ in one region of the biochip and a non-specific anti-Angiopoietin 2 (R\&D Systems) antibody (a-Ang2) $(20 \mu \mathrm{g} / \mathrm{ml}, 20 \mu \mathrm{l})$ in the other region, as a negative control. After a washing step with D-PBS $1 \mathrm{X}$ the chip was blocked with BSA (10 mg/ml) for 1 $\mathrm{h}$ at room temperature.

Immediately before use the surface of the biochips was treated with a regeneration solution made of $100 \mathrm{mM}$ glycine (Sigma-Aldrich) and $\mathrm{HCl}$ with a $\mathrm{pH}$ of 2.0 at ambient temperature. This step improves the recovery of surface reactivity after the blocking step in BSA, because any adlayers should be removed. All reagents such as ethanol (99.8\%), sulfuric acid (95\%), 30\% hydrogen peroxide solution and phosphate buffer saline (D-PBS 1X, pH 7.4) were obtained from SigmaAldrich and were used as it is.

Finally the biochip is rinsed with D-PBS $1 \mathrm{X}$ buffer again, is topped with the microfluidic element and is mounted mounted via a temperature controlled support in the optical system.

\subsection{Label-Free assay}

Once the biochip is mounted on the optical system the microfluidic channel is filled with D-PBS $1 \mathrm{X}$ and the position of the BSW resonance is tracked as a function of time in all positions along the illuminated strip. All injection and recirculation steps are performed at a flow rate of $5.5 \mu \mathrm{l} / \mathrm{s}$. After acquiring the signal for a convenient time interval to be used as a baseline, we started injecting the OVA solutions in D-PBS $1 \mathrm{X}$ according to the following procedure: 1) Washing with $500 \mu \mathrm{l}$ D-PBS 1X; 2) OVA sample injection $(60 \mu \mathrm{l})$; 3) recirculation of the solution by backward/forward pumping $(50$ cycles of $30 \mu \mathrm{l})$; 4) Washing with $1000 \mu \mathrm{l}$ D-PBS $1 \mathrm{X}$; 5) Regeneration with 100mM Glycine-HCl pH 2.0. We repeated the procedure for the following OVA concentrations in D-PBS 1X: 0.7 nM, 2.1 nM, 6.3 nM, 18.9 nM, 56.7 nM, $170 \mathrm{nM}$ and $467 \mathrm{nM}$. Such molar concentrations correspond to the following weight concentrations: $0.03 \mu \mathrm{g} / \mathrm{ml}, 0.09 \mu \mathrm{g} / \mathrm{ml}$, $0.27 \mu \mathrm{g} / \mathrm{ml}, 0.81 \mu \mathrm{g} / \mathrm{ml}, 2.43 \mu \mathrm{g} / \mathrm{ml}, 7.29 \mu \mathrm{g} / \mathrm{ml}$ and $20 \mu \mathrm{g} / \mathrm{ml}$.

Figure 4 presents the kinetic curves obtained after injecting OVA at three concentrations $(0.03 \mu \mathrm{g} / \mathrm{ml}, 0.27 \mu \mathrm{g} / \mathrm{ml}$, $2.43 \mu \mathrm{g} / \mathrm{ml}$ ). The angular position of the BSW resonance measured in CCD pixel ( 1 pixel $=0.856 \mathrm{mdeg})$ is plotted as a function of time. The kinetic signals measured in two different spots in the a-OVA region of the biochip are shown. In both cases the reference signals measured in two separate spots of the a-Ang2 negative control region on the biochip were subtracted. We observe that the signal response increases with the injected OVA concentrations. At the end of the OVA recirculation and after washing with D-PBS $1 \mathrm{X}$ the signal decreases to a stable residual value that is related to the amount of OVA captured at the surface.

In Figure 5, we show the residual BSW resonance shift (in CCD pixels) after OVA injection and washing with buffer, for two different OVA concentrations $(7.29 \mu \mathrm{g} / \mathrm{ml}$ and $20 \mu \mathrm{g} / \mathrm{ml})$. The surface of the biochip was probed by the illumination beam in 108 spots. The spots from 0 to 50 are in the a-OVA region, those from 51 to 64 are in the hydrophobic marker region, and those from 65 to 107 are in the a-Ang2 negative reference region. The residual shift is homogeneous in the two regions and the average value is larger in the signal region with respect to the negative reference.

The signals measured during the assays in all spots, and similar to those shown in Figure 4 for just two spots, were averaged over several spots in the signal (PtG/a-OVA) and in the reference region (PtG/a-Ang2). The number of spots in the averaging varies between 14 and 40, being around 30 for the majority of the data sets. Then the average signals obtained for the signal and reference regions were subtracted to compensate for any drift. 


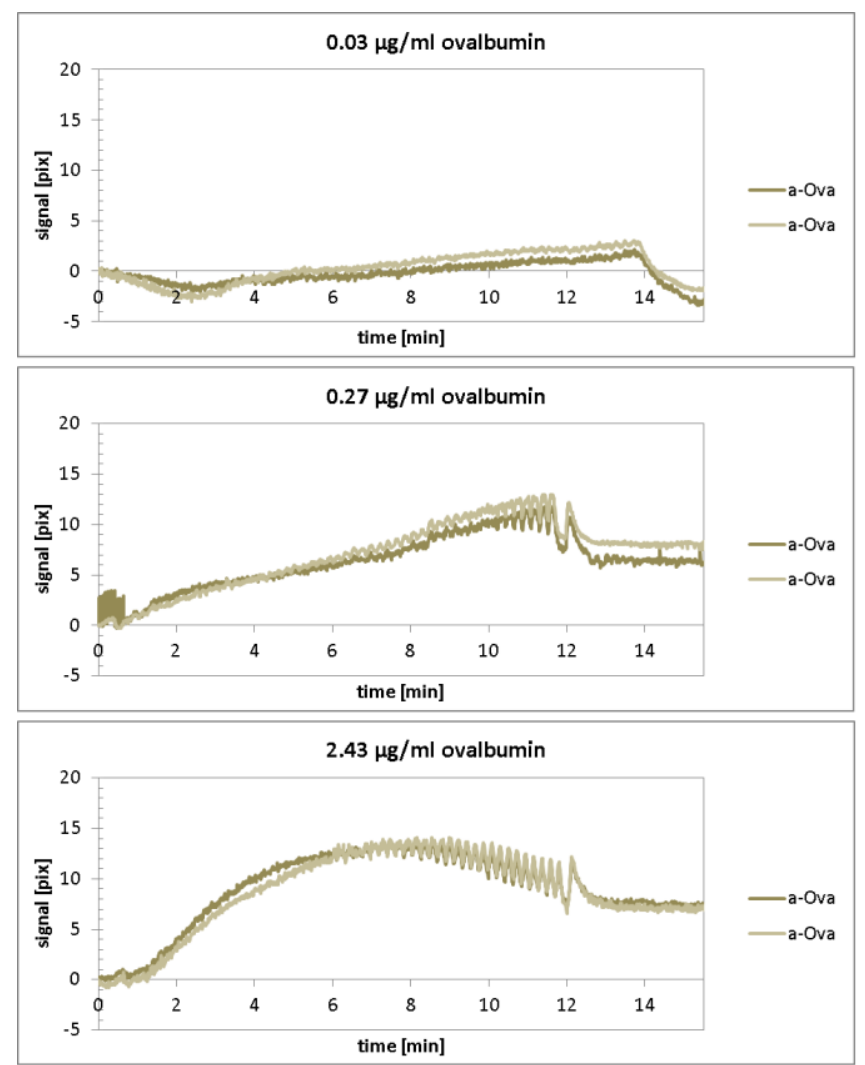

Figure 4 - Binding kinetic curves for 3 different Ova concentrations $(0.03 \mu \mathrm{g} / \mathrm{ml}, 0.27 \mu \mathrm{g} / \mathrm{ml}, 2.43 \mu \mathrm{g} / \mathrm{ml})$. For each concentration the signals measured in two spots in the a-OVA region of the chip are shown (light and dark grey curves).

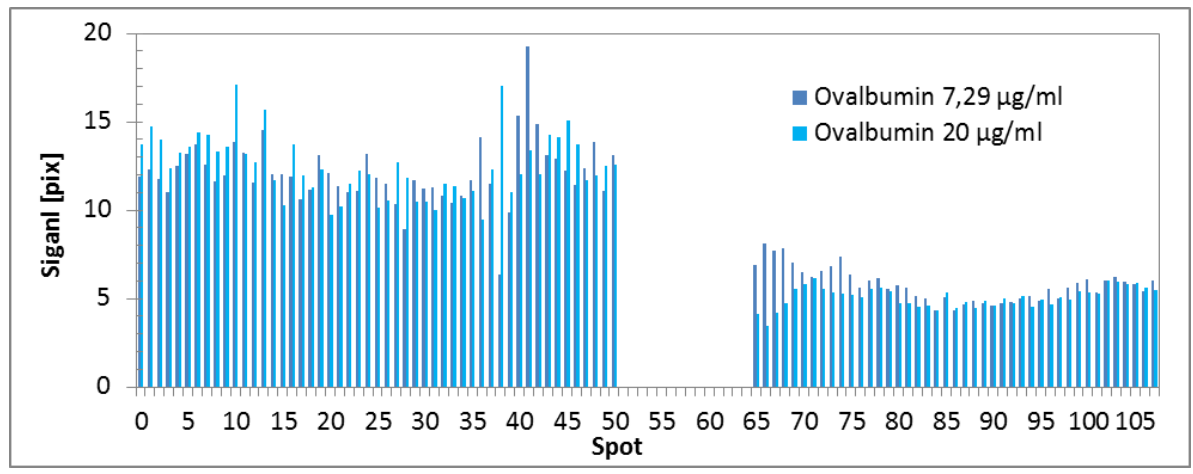

Figure 5 - BSW resonance residual shift after incubating OVA at two different concentrations, $7.29 \mu \mathrm{g} / \mathrm{ml}$ and $20 \mu \mathrm{g} / \mathrm{ml}$. The signals were measured in 108 spots along the illuminated surface of the biochip. The right region $(0,50)$ is with PtG/a-OVA and the left region $(65,107)$ is the reference with PtG/a-Ang2. The central region corresponds to the hydrophobic markers region were the BSW resonance is absent.

In all the assays carried out with the a-OVA/OVA system we observed that the regeneration procedure does not remove proteins/complexes from the biochip surface. Therefore, it is likely that the residual signals found in Figure 4 after D-PBS $1 \mathrm{X}$ washing are additive. In Figure 6 we show a plot the cumulated residual shift of the BSW resonance as a function of the concentration of the respective OVA solution. The green dots (light grey in black and white) correspond to the cumulated residual shift after washing with D-PBS $1 \mathrm{X}$ and the blue dots (dark grey in black and white) were calculated 
summing the resonance shift at the end of the recirculation for the given concentration to the residual shift for the concentration injected before.

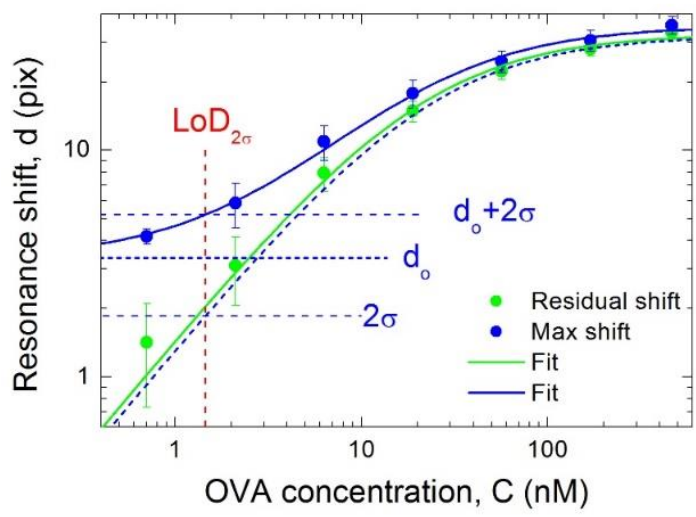

Figure 6 - Cumulated label-free signals extracted from measurements for all OVA concentrations, similar to those shown in Figure 4. The green dots (light grey) correspond to the cumulated residual shift after washing with PBS. The blue dots (dark grey) were calculated summing the mean maximum shift for the given concentration to the residual shifts for the concentrations injected before.

In Figure 6, the cumulated label-free signal at the end of the incubation of OVA concentrations (blue/dark grey dots) can be fitted to the model $d=d_{0}+d_{\max } /\left(1+\left(\mathrm{K}_{\mathrm{A}} \mathrm{C}\right)^{-1}\right)$ (blue/light grey curve), with values for the parameters reported in Table 1 . The $d_{\max }$ and $\mathrm{K}_{\mathrm{A}}$ get the same values (within the error) as those obtained by fitting the concentration dependence of the cumulative residual shift (green/light grey dots) to the model $d=d_{\max } /\left(1+\left(\mathrm{K}_{\mathrm{A}} \mathrm{C}\right)^{-1}\right)$ (green/light grey curve).

The blue (dark grey) fitting curve in Figure 6 confirms that the binding process follows the expected behaviour for a Langmuir isotherm ${ }^{19}$. However the presence of a large non-zero $\mathrm{d}_{0}$ distorts the curve in the limit of small concentrations, indicating the presence of a parasitic signal due to non-specific binding at the biochip surface. Probably such behaviour is due to an insufficient blocking of the biochip surface that adsorbs OVA biomolecules not-specifically in open binding sites. This result suggests that care should be taken to block the biochip surface with the aim to reduce $d_{0}$ as much as possible, ideally down to zero.

Table 1. - Values of the parameters used to fit the concentration dependence of the cumulated label-free for the Ova assay.

\begin{tabular}{|c|c|}
\hline Parameter & Value \\
\hline$d_{\max }($ pix $)$ & $32.0 \pm 1.9$ \\
\hline $\mathrm{K}_{\mathrm{A}}\left(\mathrm{M}^{-1}\right)$ & $(4.24 \pm 0.98) \times 10^{7}$ \\
\hline$d_{0}(\mathrm{pix})$ & $3.34 \pm 0.71$ \\
\hline
\end{tabular}

From the analysis of the data reported in Figure 6 we can determine the limit of detection of the platform when operated in the label-free mode $\left(\mathrm{LoD}^{\mathrm{LF}}\right)$ for the a-OVA/OVA system. Once $\mathrm{d}_{0}$ is determined, we can assert that a signal can be detected if it differs from $\mathrm{d}_{0}$ by more than twice the standard deviation $(\sigma)$ of its associate measurement error. From the equality $d_{\max } /\left(1+\left(\mathrm{K}_{\mathrm{A}} \mathrm{C}\right)^{-1}\right)=2 \cdot \sigma$, we then find $\mathrm{LoD}^{\mathrm{LF}}=1.45 \mathrm{nM}$, corresponding to $60 \mathrm{ng} / \mathrm{ml}$.

\subsection{Fluorescence assay}

From the analysis of the label-free experiments reported above it is clear that, for low OVA concentrations, the signals are affected by large errors due to not perfect compensation of parasitic effects (non-specific binding, local temperature and pressure effects, functionalization and immobilization inhomogeneities). The signals are immersed in a relatively large noise that does not permit to infer any information on OVA binding events. This limits the $\mathrm{LoD}^{\mathrm{LF}}$ as shown above. In this 
respect the complementary fluorescence assay permits to decrease of the limit of detection and discloses information on the OVA binding process.

As an example in Figure 7 (left) we show the result of a label-free assay carried out at an OVA concentration of $30 \mathrm{ng} / \mathrm{ml}$. With respect to the measurements reported in the Section 4.2 here in the reference spot we immobilized anti-Human Serum Albumin (a-HSA) as a non-specific antibody ( $8 \mu \mathrm{M}$ for 1 hour at ambient temperature). The curve is the difference between the averaged BSW minima positions in the signal and reference regions ( 22 and 42 spots of the a-OVA and a-HSA regions, respectively).

Starting from $\mathrm{t}=0 \mathrm{~min}$, the assay was carried out according to the following protocol: 1$)$ OVA injection (100 $\mu 1$ at 30 $\mathrm{ng} / \mathrm{ml})$; 2) Backward/forward recirculation (50 cycles of $25 \mu \mathrm{l})$; 3) Washing twice with $500 \mu \mathrm{l}$ PBS; 4) Background Fluorescence measurement (integration time $1 \mathrm{~ms}$ ); 5) Washing with $500 \mu \mathrm{l} \mathrm{PBS}$; 6) Biotin-Labelled a-OVA* injection (100 $\mu \mathrm{l}$ at $10 \mu \mathrm{g} / \mathrm{ml})$; 7) Backward/forward recirculation (50 cycles of $25 \mu \mathrm{l})$; 8) Washing twice with $500 \mu 1$ PBS; 9) Labelled neutravidin (Dylight-650) injection $(100 \mu \mathrm{l}$ at $10 \mu \mathrm{g} / \mathrm{ml}) ; 10)$ Backward/forward recirculation $(50$ cycles of 25 $\mu 1)$; 11) Washing with $500 \mu \mathrm{l}$ PBS; 12) Fluorescence Measurement (integration time $1 \mathrm{~ms})$. All injections were carried out at a flow speed of $5.5 \mu 1 / \mathrm{s}$.

In Figure 7 (left) the time origin is set to the moment of injection of $100 \mu \mathrm{l}$ of OVA solution. The various injections take place during the time intervals: $(0,9 \mathrm{~min})$ OVA; (18, $27 \mathrm{~min})$ biotinylated detection a-OVA*; (32, 41 min) Dylight650 labelled neutravidin. Fluorescence emission was measured in two time intervals: (12, 16 min) before labelling, (46, 50 min) after labelling with Dylight-650.
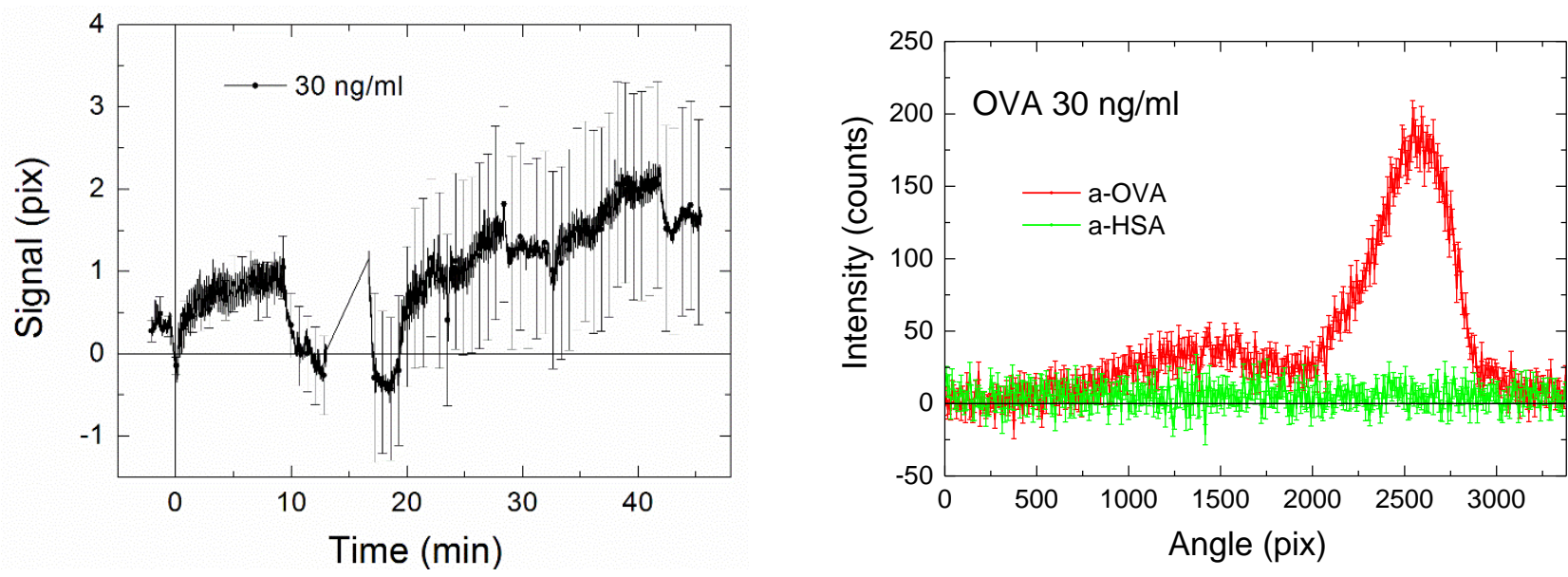

Figure 7 - (left) Kinetics of the difference of the averaged BSW resonance angles in the signal and reference regions (22 spots of the a-Ova region and 42 spots of the a-HSA region) upon injection of Ova at $30 \mathrm{ng} / \mathrm{ml}$, biotinylated detection a-Ova at $10 \mu \mathrm{g} / \mathrm{ml}$, and Dylight-650 labelled neutravidin at $10 \mu \mathrm{g} / \mathrm{ml}$. (right) Angular distribution of the background subtracted fluorescence emission in the reference (a-HSA) region (green) and in the signal (a-Ova) region (red).

As it can be seen the difference signal goes back to the starting level after injection of OVA at $30 \mathrm{ng} / \mathrm{ml}$ and subsequent washing with D-PBS 1X ( $\mathrm{t} \sim 12 \mathrm{~min})$. Such result confirms that the concentration $30 \mathrm{ng} / \mathrm{ml}$ is below the limit of detection of the label-free operation mode (for the used surface chemistry and protein immobilization procedures). After performing the second part of the assay, $t \in(17,46 \mathrm{~min})$, the label-free signal is enhanced due to the mass loading effect when the detection antibody and the neutravidin bind to the OVA captured at the surface. One can observe a differential shift of the BSW resonances during the incubation of the species and a residual shift of about 1.5 pixel. However, the uncertainty of the measurement is still too large to claim label-free detection.

In Figure 7 (right) we show the result of the fluorescence measurement for the same OVA assay at $30 \mathrm{ng} / \mathrm{ml}$. The curves represent the angular distributions of the background subtracted fluorescence emission in the reference (a-HSA) region (green) and in the signal (a-OVA) region (red). The curves were obtained by averaging 20 spots in either the reference or 
in the signal region. As it can be seen the fluorescence intensity in the signal region is well distinguished from the intensity in the reference region.

If we assume that for very low concentrations the density of bound OVA biomolecules, and therefore the fluorescence signal scales linearly, then we can estimate the limit of detection of the platform in the fluorescence mode:

$$
L o D^{F L U O}=30 \mathrm{ng} /\left.\mathrm{ml} \cdot \frac{\text { Average Intensity Counts }(\text { Anti }-H S A)}{\text { Average Intensity Counts }(\text { Anti }- \text { OVA })}\right|_{30 \mathrm{ng} / \mathrm{ml}}
$$

Extracting data from Figure 7 (right) one gets $\mathrm{LoD}^{\mathrm{FLUO}}=3.8 \pm 0.5 \mathrm{ng} / \mathrm{ml}$ corresponding to $0.08 \pm 0.01 \mathrm{nM}$. Such results demonstrate that the implementation of the fluorescence detection, not available in the SPR case, can lead to an improvement of the limit od detection, that is at least 10 times smaller than what found in the label-free mode.

\section{CONCLUSIONS}

The present work describes an approach based on BSW to combine label-free and fluorescent labels detection in a single biosensing platform.

Low loss one dimensional photonic crystals featuring narrow surface wave resonances have been designed and fabricated on injection molded chips. A sandwich assay that detects ovalbumin has been successfully demonstrated. The tracking of the angular resonance position yields a label-free detection with $\mathrm{LoD}^{\mathrm{LF}}=60 \mathrm{ng} / \mathrm{ml}$. This result is supported by the clear difference of fluorescence intensities obtained on measurement and reference spots, indicating a much lower limit of detection $\mathrm{LoD}^{\mathrm{FLUO}}=3.8 \mathrm{ng} / \mathrm{ml}$.

As a whole, successful combination of label-free and fluorescence modes in one optical detection system is achieved. Notably, the combination of angular and wavelength resolution in fluorescence mode yields new parameters for read-out that opens up new possibilities for designing different assays. The complete technological platform constituted by the instrument and the biochips is available for large scale production thus making the chip approach a valid candidate for disposable biosensor application.

\section{ACKNOWLEDGEMENTS}

This work was supported in part by the European Commission through the project BILOBA (www.biloba-project.eu; Grant agreement 318035).The authors gratefully acknowledge Thomas Schubert from KDS Radeberger Werkzeugbau GmbH (Germany) for the TOPAS ${ }^{\circledR}$ substrates manufacturing and Prof. Federico Bussolino from the University of Torino (Italy) for fruitful discussions.

\section{REFERENCES}

[1] Yeh, P., Yariv, A. and Hong, C.-S., "Electromagnetic propagation in periodic stratified media. I. General theory,” J. Opt. Soc. Am. 67(4), 423-438 (1977)

[2] Robertson, W. M. and May, M. S., "Surface electromagnetic wave excitation on one-dimensional photonic bandgap arrays," Appl. Phys. Lett. 74(13), 1800-1802 (1999)

[3] Shinn, M. and Robertson, W. M., "Surface plasmon-like sensor based on surface electromagnetic waves in a photonic band-gap material," Sens. Act. B 105, 360-364 (2005)

[4] Konopsky, V. N. and Alieva, E. V., "Photonic crystal surface wave for optical biosensors," Anal. Chem. 79(12), 4729-4735 (2007)

[5] Sinibaldi, A., Danz, N., Descrovi, E., Munzert, P., Schulz, U., Sonntag, F., Dominici, L. and Michelotti, F., „Direct comparison of the performance of Bloch surface wave and surface plasmon polariton sensors," Sens. Act. B 174, 292-298 (2012) 
[6] Sinibaldi, A., Rizzo, R., Figliozzi, G., Descrovi, E., Danz, N., Munzert, P., Anopchenko, A. and Michelotti, F., “A full ellipsometric approach to optical sensing with Bloch surface waves on photonic crystals," Opt. Expr. 21(20), 23331-23344 (2013)

[7] Rizzo, R., Danz, N, Michelotti, F., Maillart, E., Anopchenko, A. and Wächter, C., "Optimization of angularly resolved Bloch surface wave biosensors," Opt. Expr. 22(19), 23202-23214 (2014)

[8] Ballarini, M., Frascella, F., Michelotti, F., Digregorio, G., Rivolo, P., Paeder, V., Musi, V., Giorgis, F. and Descrovi, E., "Bloch surface waves-controlled emission of organic dyes grafted on a one-dimensional photonic crystal," Appl. Phys. Lett. 99(4), 043302 (2011)

[9] Sinibaldi, A., Fieramosca, A., Rizzo, R., Anopchenko, A., Danz, N., Munzert, P., Magistris, C., Barolo, C., and Michelotti, F., "Combining label-free and fluorescence operation of Bloch surface wave optical sensors," Opt. Lett. 39(10), 2947-2950 (2014)

[10] Danz, N., Kick, A., Sonntag, F., Schmieder, S., Höfer, B., Klotzbach, U. and Mertig, M., „Surface plasmon resonance platform technology for multi-parameter analyses on polymer chips,“"Eng. Life Sci. 11(6) 566-572 (2011)

[11] Danz, N., Sinibaldi, A., Munzert, P., Anopchenko, A. Förster, E., Schmieder, S., Chandrawati, R., Rizzo, R., Heller, R., Sonntag, F., Mascioletti, A., Rana, S., Schubert, T., Stevens, M.M. and Michelotti, F., „Biosensing platform combining label-free and labelled analysis using Bloch surface waves, " Proc. of SPIE, 9506, 95060V (2015)

[12] Raether, H. „Surface Plasmons on smooth and rough surfaces and on gratings, “ Springer Tracts in Modern Physics, vol. 111, Ed. Berlin: Springer-Verlag,, (1988)

[13] Sinibaldi, A., Danz, N., Anopchenko, A., Munzert, P., Schmieder, S., Chandrawati, R., Rizzo, R., Rana, S., Sonntag, F., Occhicone, A., Napione, L., De Panfilis, S., Stevens, M.M. and Michelotti, F., „Label-free detection of tumor angiogensis biomarker Angiopoietin 2 using Bloch surface waves on one dimensional photonic crystals, " Jour.Lightwave Tech., 33(16), 3385-3394 (2015)

[14] Munzert, P., Schulz, U. and Kaiser, N., „Transparent thermoplastic polymers in plasma assisted coating processes,“ Surf. Coat. Tech., 174-175, 1048-1052 (2003)

[15] Sinibaldi, A., Anopchenko, A., Rizzo, R., Danz, N., M,unzert, P., Rivolo, P., Frascella, F., Ricciardi, S., and Michelotti, F., „Angularly resolved ellipsometric optical bio-sensing by means of Bloch surface waves, “ Anal. \& Bioanal. Chem., 407(14), 3965-3974 (2015)

[16] Danz, N., Waldhäusl, R., Bräuer, A. and Kowarschik, R., „Dipole lifetime in stratified media,“ J. Opt. Soc. Am. B 19(3), 412-419 (2002)

[17] Flämmich, M., Frischeisen, J., Setz, D. S., Michaelis, D., Krummacher, B. C., Schmidt, T. D., Brütting, W. and Danz, N., "Oriented phosphorescent emitters boost OLED efficiency," Org. Electron. 12, 1663-1668 (2011)

[18] see „DyLight $\AA \quad$ Fluorochrome $\quad$ conjugated $\quad$ secondary http://www.abcam.com/index.html?pageconfig=resource\&rid=12934

[19] O’Shannessy, D. J. and Winzor, D. J. „Interpretation of Deviations from Pseudo-First-Order Kinetic Behavior in the Characterization of Ligand Binding by Biosensor Technology, “ Analytical Biochemistry 236, 275-283 (1996) 